\title{
CLASSIFICATION OF RHEUMATISM
}

\author{
By KENNETH STONE, D.M.Oxon., M.R.C.P.
}

\section{(Assistant Physician, B.R.C.S. Clinic for Rheumatism, Peto Place, N.W.I; Consultant in Physical Medicine, E.M.S.)}

"The arrangement of things according to their resemblances or identities"-Jevons' definition of classification-has played a small though necessary part in the growth of biology from the time when rules of classification were first laid down by Aristotle. It is a useful contrivance for the acquisition of elementary knowledge, and therefore is still relevant to the study of Rheumatism.

\section{LOGICAL CLASSIFICATION}

A classification may be either artificial or natural. The first serves some immediate practical purpose. Thus I could classify rheumatic diseases by arranging them in alphabetical order, which might be useful for indexing and reference. But it is clear that in any artificial classification the things grouped together are likely to have very few properties in common; diseases whose names begin with A or B will almost certainly have no other common characters.

The essence of natural classifications is that the things grouped together share a large number of common properties. Jevons writes optimistically that "they give us the deepest resemblances and relations, and may lead ultimately to a knowledge of the way in which the variety of things are produced."

There are several rules to which a sound natural classification must conform. It must, for example, be exhaustive; that is, it must include all things which properly belong to the group, and none which does not. It follows that I must be able to define the group of objects I am classifying. This necessity is imposed by another requirement, that each class in the division must be defined; for I can only define a thing if I can first define its next higher genus. There has been a curious reluctance to attempt a definition of the Rheumatic Diseases.

Perhaps the most important rule is that the division of a genus must be grounded upon one principle, or basis. Though regarded by modern logicians as a counsel of perfection, it cannot be just ignored. If I wish to classify 'doctors', I can divide them first into physicians, surgeons, obstetricians, and so on, the principle of division being the type of work which they do. Alternatively, I can divide them by nationality, or by personality, or success. But if I mix these principles in the same division, it might turn out: physicians, surgeons, Welshmen, critics, rogues, and the Editor of the Lancet - a result which is sheer confusion.

Just this type of confusion is achieved in the well-known classification evolved by a nomenclature sub-committee of the British Committee on Rheumatic Diseases appointed by the Royal College of Physicians in I934. Using three principles-clinical, pathological, and anatomicalthey arrive at four major groups of Rheumatism: I. Rheumatic fever, acute or sub-acute; II. Acute gout; III. Chronic arthritis; IV. Non-articular rheumatic affections. Having begun with a clinical basis, a clinical basis should have been used to the end of that first division. When each sub-group is treated as a genus and further divided, the same rule applies; I must use either the same or a new principle, and again one only for each group.

Another rule broken by this classification is that one must not proceed at once from a wide genus to a narrow species. I cannot appropriately divide 'Englishmen' into those who are Rheumatologists and those who are not; it is equally bad to make acute gout a major group of rheumatism.

This illustrates the more general rule that the principle of division must be appropriate. The feature which distinguishes a natural from an artificial classification is that the principle of 
division is a quality from which it is possible to infer, in those things which possess it, a large number of other qualities.

Thus if I choose a pathological basis of division, say inflammation, it is likely that I shall be able to infer a large number of other facts about disorders characterised by an inflammatory tissue response.

On the other hand, if I take as a basis of division the property of having 'known associated factors' - as in the Royal College of Physicians' classification-I choose a quality from which scarcely anything can be inferred. The result is the appearance in one group of climacteric arthritis, gout, and multiple infective arthritis, diseases which are as dissimilar as sciatica, cerebral haemorrhage, cancer and'gastric ulcer, which also'have known associated factors. The essence of a natural classification is that things shall appear together which have deep and fundamental resemblances.

\section{LOGICAL RULES APPLIED TO THE CLASSIFICATION OF RHEUMATISM}

Definition of rheumatism. The most satisfactory way to define anything capable of classification is, in the words of formal logic, to give the proximate gentus and the 'difference'that is, by stating the essential characters of the next higher genus, and the special characters which distinguish the object we are defining from others of the same genus. Thus I can define 'tubercle bacillus' if I can first define 'acid-fast bacillus', and state the properties which distinguish the tubercle bacillus from the smegma bacillus and the bacillus of leprosy.: Similarly I can define. 'acid-fast bacillus' if I can first define 'bacillus'.

Can we say that the proximate genus of rheumatism is 'all disorders of the locomotor system'?

Not until the I8th century was any distinction made between different forms of joint trouble. Before that time rheumatism, gout and arthritis were almost synonymous, being loosely used to cover any affection of the joints. Gradually what we know as gout was distinguished from other joint disorders; rheumatism' lingered on as a name for these, and was also used to denote obscure painful conditions of the muscles, whether associated with arthritis or not. It seems to have been regarded as a specific disease, affecting mainly joints or muscles, and capable of affecting the heart.

The history of the word would thus support its use as a name for a class within the larger group of locomotor system disorders. If it be accepted that this is the proximate genus, what particular characters distinguish rheumatism from all other disorders of the locomotor system?

I have never been able to determine whether the original idea of an essential etiological unity in the rheumatic diseases is still current to-day. In the case of rheumatoid arthritis and its variants, it is a tenable hypothesis that there is a specific materies morbi. It could be that we are dealing with a specific virus, perhaps a latent virus living in perfect symbiosis with its host, until under the stimulus of cold, or fatigue, or focal sepsis, or parturition, or what-not, it becomes aggressive and pathogenic. But if it be held that the same materies morbi is also responsible for osteo-arthritis, climacteric arthritis, fibrositis, bursitis, tenosynovitis, neuralgias, and all other disorders classed as rheumatic in monographs which treat of the subject, I can only say that in the whole range of human pathology no other materies morbi is quite so versatile, nor any other disease so erratic.

But if we can find no common etiological characters which would serve as a criterion, in what sense are we using the name rheumatism? I would suggest that to single them out from all other disorders of the locomotor system by two features which they have in common would provide a definition in line with common usage. The first is an obscure etiology. We no 
longer call a disorder rheumatic when etiology is clearly revealed. When an inflamed joint is proved to be caused by the gonococcus, tact alone justifies us in calling it rheumatism. The second common feature is pain.

\section{I submit then that we might define rheumatism as painful disorders of the locomotor system whose etiology is obscure.}

There are two corollaries. When the last rheumatic disease has yieldęd up its secrets, 'rheumatism' wtll disappear from medical nomenclature. The disorders will still be there as disorders of the locomotor system; and the 'rheumatologist' one hopes will stay with them, perhaps now more happily called by some other name.

Again, if this definition of rheumatism be accepted, it is of doubtful value to attempt a natural classification at all. It would be more far-sighted to classify all disorders of the locomotor system whether of known or unknown etiology, in the hope of bringing out relationships between the obscure disorders and those which are less obscure. There is little doubt that progress usually comes by proceeding from the known to the unknown, and the tendency to separate rheumatic diseases from diseases of muscles and joints (or what may perhaps be called orthopedic medicine) is as little intelligible as would be the separation from neurology of all obscure nervous diseases.'

By the word 'disorder' in this definition, I mean not only diseases of the locomotor system, or whose impact is primarily on the locomotor system, such as tuberculosis of a joint, gout, or rheumatoid arthritis; but also manifestations of disease whose impact is chiefly elsewhere, such as the joint reactions of rheumatic fever or german measles, and morbid tissue changes, such as loose bodies in joints and many examples of the so-called osteo-arthritis.

For the first group I have used the term 'disease.' A disease is the total unified evolving pattern of cause and effect, which is manifest by disturbance of physiological function. It is an event, and therefore a reality in the sense in which Whitehead uses these terms. By disease of a system, such as the locomotor system, I mean that the central feature of the event is a disturbance of function of that system.

On the other hand, the second group comprises names which stand for symptoms, disturbances of function, or morbid tissue changes. They are no more than ingredients of the total event which is a disease, torn from their context.' 'Myalgia' may be a bit of the pattern of a general toxic state, or of peripheral vascular disease; 'neuralgia' of herpes, or a disc lesion; 'osteo-arthritis' of chronic strain, recurrent synovitis, gout, or any disease which over a long period alters the normal stresses and strains within a joint.

I have called these fragments of disease 'component syndromes.' 'Syndrome' means a concurrence, although for no known reason it is popularly applied only to a concurrence of symptoms, The qualifying 'component' indicates a concurrence which is an ingredient of a total pattern.

Clearly a component syndrome is an abstraction; it is not possible for the body to be the scene of a fragment of a disease. Abstractions are not classifiable, unless artificially. A bibliographer could make a natural classification of old manuscripts, but only an artificial one of fragments torn from them.

This broad distinction into diseases and component syndromes is fundamental; it is a first step in any future rheumatology.

In the following classification Table I is of diseases; diseases of the locomotor system of known etiology have been included, for reasons given earlier (the rheumatic diseases are shown in heavy type). In Table II only component syndromes which by definition would properly be called rheumatic have been enumerated.

Knowledge is as yet too elementary to make any final grouping. I should not be unduly disturbed by the transfer of any disorder from one group to another. Perhaps fibrositis should appear in Table II. If this classification has any merit, it can only lie in the principles which guided its construction. 
Diseases of the locomotor system

\section{TABLE I}

A. OF JOINTS AND THEIR ADNEXA.

I. TISSUE REACTION ONE OF INFLAMMATION

I.I Infective type of inflammatory response

I.II Pyogenic type

Streptococcal, staphylococcal,

gonococcal arthritis

I.I2 Tuberculous type

Tuberculous arthritis

I.13 Rheumatoid type

Rheumatoid arthritis

variants-

Atypical rheumatoid arthritis

Still's disease

Spondylitis

I.2 Chronic traumatic type of inflammatory response

Chronic synovitis

Cartilage injuries .

Strains and Sprains, etc.

I.3 Allergic'type of inflammatory response

Acute gout

Purpura rheumatica, and other forms of 'allergic' polyarthritis

? Intermittent hydrarthrosis

I.4 Type of inflammatory response unknown

Capsulitis of the shoulder

Bursitis and tenosynovitis.

Chronic gout

2. TISSUE REACTION ONE OF DEGENERATION

Intervertebral disc lesions

B. OF FIBROUS TISSUES

\section{Fibrositis}

C. OF. THE NEURO-MUSCULAR SYSTEM

None which by definition would be properly called Rheumatic

TABLE II

Component syndromes (subject only to artificial classification)

A. PATHOLOGICAL TISSUE CHANGES

Osteo-arthrosis

Spondylosis

Menopausal arthrosis of the knee

Inflamed joints associated with Rheumatic fever, Influenza,

Rubella, and other specific general infections

B. SYMPTOMS

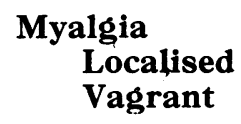

Neuralgia

Intercostal

Brachial

Sciatic 